\title{
A Review on Scream Classification for Situation Understanding
}

\author{
Saba Nazir, Muhammad Awais \\ Dept. of Software Engineering \\ Govt. College University \\ Faisalabad, Pakistan
}

\author{
Sheraz Malik \\ Dept. of Information Technology \\ Govt. College University \\ Faisalabad, Pakistan
}

\author{
Fatima Nazir \\ Dept. of Software Engineering \\ Govt. College University \\ Faisalabad, Pakistan
}

\begin{abstract}
In our living environment, a non-speech audio signal provides a significant evidence for situation awareness. It also compliments the information obtained from a video signal. In non-speech audio signals, screaming is one of the events in which the people like security guard, care taker and family members are particularly interested in terms of care and surveillance because screams are atomically considered as a sign of danger. Contrary to this concept, this review is particularly targeting automated acoustic systems using non-speech class of scream believing that the screams can further be classified into various classes like happiness, sadness, fear, danger, etc. Inspired by the prevalent scream audio detection and classification field, a taxonomy has been projected to highlight the target applications, significant sound features, classification techniques, and their impact on classification problems in last few decades. This review will assist the researchers for retrieving the most appropriate scream detection and classification technique and acoustic parameters for scream classification that can assist in understanding the vocalization condition of the speaker.
\end{abstract}

Keywords-Scream classification; scream detection; acoustic parameters; surveillance; security

\section{INTRODUCTION}

In the past few decades, there have been several efforts regarding the classification of the acoustic data into classes. The audio data is very informative and a rich source of extraction for the type of content involving content-based classification of the acoustic signals. Human beings use vocal tract for producing speech sounds such as talking, singing, crying, and laughing. These sounds are further classified as speech or non-speech vocalizations. Speech consists of voices that are in the form of sentences and can be understood using different Natural Language Processing (NLP) techniques. The non-speech sounds include laugh, sneeze, cough, snore, and scream. These non-speech vocalizations are sometimes segregated from speech signals to extract additional information about the context, situation, or emotional state of the speaker. Scream is a non-speech signal that is caused by a loud vocalization when air passes through vocal folds with greater force than regular vocalizations. Most often, a scream is a reflex action or a response from an unexpected situation and it is strongly associated with emotional behavior of the speaker. It can have many forms like a scream of joy, danger, pain, surprise, etc.

Scream sound event classification and detection has wide applications in science due to which it has gained significant importance in literature. Many real-life acoustic systems use scream detection in the areas like speaker identification [1], Audio-Surveillance Systems [2] and Home applications [3]. These systems use the knowledge extracted from scream detection and classification for processing. In this field, the conjunction of time-frequency features and machine learning classifier have achieved recent developments. Different techniques and methodologies have been established to differentiate speech and non-speech sounds. These include Support Vector Machines [3], band-limited spectral entropy [4], Deep Neural Networks (DNN) [5], Hidden Markov Model (HMM), sound event partitioning [6] and modulation power spectrum [7].

Most works on scream detection and classification emphasize on some crucial acoustic events, none cover the overall state-of-the-art for scream classification and detection. The current work varies of all preceding efforts in terms of emphasis, correctness as well as suitability. The aim of this review is to highlight the scream classification concerns and challenges to analyze and classify the screams from a variety of perspectives. Additionally, a comparative study is hereby presented that is based on the problem domain, sound features, and classification techniques. By overviewing this review, one can easily determine the problem domains where to put the scream efforts, using best sound parameters and scream classification techniques for situation understanding.

This review is planned as follows. Section 2 covers the data collection techniques and research methodology. Section 3 contains an overview of different classes of problem domains, sound features, and classification techniques. Section 4 evaluates the various data classes and argues on the comparison and accuracy rates. Finally, Section 5 concludes the key points in this review.

\section{DAta Collection}

A review of 30 different research articles that are associated with scream detection and classification in various environments is presented. Highly cited and credible publications are used from different digital libraries for obtaining the research source. A thorough analysis is performed on all the articles to make sure that the content is pertinent to the research interests. Those classification problems that have hindered the further development and exploration in screaming environments, are discussed. 


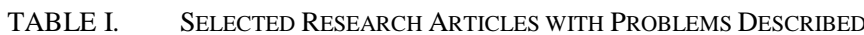

\begin{tabular}{|c|c|c|c|c|}
\hline \# & Name/ Ref & Year & Problem & Detection/ Classification \\
\hline 1. & A. Pillai et al.[8] & 2018 & Classifying violent extensive audios like music, speech, gunshots, and screams. & Detection \\
\hline 2. & J. H. L. Hansen et al.[1] & 2017 & Analyzing human screams for text-independent speaker identification & Detection \\
\hline 3. & N. Hayasaka et al. [4] & 2017 & Detection of human scream considering noise robustness & Detection \\
\hline 4. & S. Chung et al. [9] & 2017 & Detecting screams for social problems and violent crimes in public places & Detection \\
\hline 5. & S. Mun et al. [10] & 2017 & Classification of acoustic scene using screams & Detection \\
\hline 6. & L. Girin [5] & 2016 & Automating screams detection in subway trains & Detection \\
\hline 7. & Y. Li et al. [11] & 2016 & $\begin{array}{l}\text { Automatically classifying audio events like glass breaking, gunshots, footsteps, } \\
\text { and screams for surveillance. }\end{array}$ & Detection \\
\hline 8. & A. Sharma et al. [12] & 2016 & Scream and cry detection in urban environments & Detection \\
\hline 9. & L. H. Arnal et al.[7] & 2015 & $\begin{array}{l}\text { Using acoustic analysis, psychophysical experiments, and neuroimaging to } \\
\text { isolate screaming features, and track their processing in the human brains }\end{array}$ & Detection \\
\hline 10. & J. H. L. Hansen et al. [13] & 2015 & $\begin{array}{l}\text { Robustlly detecting screams in noisy areas using unsupervised learning } \\
\text { algorithm. }\end{array}$ & Detection \\
\hline 11. & M. Z. Zaheer et al. [14] & 2015 & Scream detection for existing CCTV cameras for better surveillance. & Detection \\
\hline 12. & M. K. Nandwana et al. [15] & 2014 & $\begin{array}{l}\text { Finding out the impact of screaming on the performance of text independent } \\
\text { speaker recognition systems }\end{array}$ & Detection \\
\hline 13. & M. Vacher et al. [16] & 2014 & Sound classification for patients and elderly people hospitalized at home. & Detection \\
\hline 14. & M. Vacher et al. [17] & 2014 & Detection and classification of acoustic events in a noisy environment & Detection \\
\hline 15. & B. Lei et al. [18] & 2014 & Power-efficient sound-event detection. & Detection \\
\hline 16. & K. Kato [19] & 2013 & Clarifying audio features of the death growl as well as screaming voice. & Detection \\
\hline 17. & B. Uzkent et al. [20] & 2012 & Classification of non-speech environmental sounds using new feature set. & Detection \\
\hline 18. & M. Mark et al. [21] & 2012 & $\begin{array}{l}\text { Investigate the consumption of power for a sound-event classification system at } \\
\text { different stages }\end{array}$ & Detection \\
\hline 19. & W. Huang et al. [3] & 2010 & $\begin{array}{l}\text { Detection of human screams using analytic and statistical features as a method } \\
\text { of classification. }\end{array}$ & Detection \\
\hline 20. & C. Chan et al. [22] & 2010 & $\begin{array}{l}\text { Scream/Non-scream classification in an abnormal situation such as bank } \\
\text { robbery }\end{array}$ & Detection \\
\hline 21. & W. Liao et al. [23] & 2009 & Analysing non-speech human sounds, like scream, laugh, snore and sneeze. & Detection \\
\hline 22. & A. Fleury et al. [24] & 2008 & Home based global speech and sound recognition system for surveillance. & Detection \\
\hline 23. & L. Gerosa et al. [2] & 2007 & $\begin{array}{l}\text { audio-based surveillance system to detect anomalous acoustic events like } \\
\text { screams or gunshots.in public. }\end{array}$ & Detection \\
\hline 24. & C. Zhang et al. [25] & 2007 & $\begin{array}{l}\text { Collectively consider the five speech modes in maintaining speech system } \\
\text { performance for coding, speech, and speaker recognition. }\end{array}$ & Detection \\
\hline 25. & A. Rabaoui et al. [26] & 2007 & Improving one-class SVM classifier for sounds classification. & Detection \\
\hline 26. & P. C. Schön et al. [27] & 2004 & Develop a system to record and monitor level of anxiety sounds/calls in pigs. & Detection/ Classification \\
\hline 27. & M. Vacher et al. [28] & 2004 & $\begin{array}{l}\text { Scream detection using transient models to ensure short detection delay in } \\
\text { medical telesurvey }\end{array}$ & Detection \\
\hline 28. & E. R. Siebert et al. [29] & 2003 & Analyzing the structure and context of chimpanzee screams. & Detection \\
\hline 29. & N. E. O. Connor et al. [30] & 2002 & $\begin{array}{l}\text { Unusual scene and sound detection in web-cam images using unsupervised } \\
\text { learning }\end{array}$ & Detection \\
\hline 30. & R. A. Breguet et al. [31] & 2000 & $\begin{array}{l}\text { Automatic detection/recognition of impulsive sounds, such as human screams, } \\
\text { glass breaks, gunshots, door slams or explosions. }\end{array}$ & Detection \\
\hline
\end{tabular}


TABLE II. DATA CLASSIFICATION

\begin{tabular}{|l|l|l|}
\hline Sr. & Type & Class \\
\hline 1. & Problem Domain & $\begin{array}{l}\text { Surveillance } \\
\text { Speaker Identification } \\
\text { Feature Enhancement }\end{array}$ \\
\hline 2. & Feature Extraction & $\begin{array}{l}\text { Temporal } \\
\text { Spectral } \\
\text { Prosodic }\end{array}$ \\
\hline 3. & Classification Techniques & $\begin{array}{l}\text { Supervised Learning } \\
\text { Unsupervised Learning }\end{array}$ \\
\hline
\end{tabular}

The selected data has been divided into different categories to carve out possible alternatives in several directions. Table I presents literature works related to the scenarios using scream detection or classification as major. The problem of each research article is described along with its ability of detection or classification of screams. Most of the authors are focusing on using the scream detection in the surveillance systems as in a common understanding the screams are a source of danger. Other have focused on whether enhancing the sound features of the systems under study or the identifying the speakers by their vocal scream samples. Only one author has worked indirectly on scream classification along with detection for animal screams.

These research studies analyze and compare the crucial aspects of different scream detection and classification methods. The major concerning factor is the accuracy of detection and classification stages, while minimizing the error rates and choosing the best possible sound features. In this review the emphasis is on the aspects of proficiency and accuracy of scream classification techniques.

On the first glance of Table I, it is very unsure to find out the loose ends and research gaps for a researcher who is new to this field. For this reason, each source is separated in terms of its problem domain, sounds parameters, type of classification technique used, and the results obtained in each case. All these categories are later further divided into different classes for even a broader understanding. Furthermore, tables and graphs are used in each class to compare on source with the other to find out which domain, parameter or technique is the best one to work out in future.

\section{DATA ClassificATION}

The process of organizing data into groups and categories for its most effective and efficient use is broadly defined as data classification. As described above the collected data samples from different sources are analyzed based on the parameters discussed in Table II.

\section{A. Problem Domain}

A problem domain is the area of knowledge or application that desires to be analyzed and examined to solve a problem. Converging on a problem domain is simply focusing at only the topics of a person's interest, and apart from everything else. Based on the observations from various research sources, the problem domain has been divided into three categories: i) Surveillance, ii) Speaker Identification, and iii) Acoustic
Features Enhancement. All these categories are discussed in detail below:

1) Surveillance: Surveillance means managing, protecting, influencing, or directing the people by monitoring the abnormal activities or changing information in their surroundings [32]. Surveillance systems enable the remote observation of prevalent society for public safety and integrity. These observations can be made by some electronic devices like audio/video recordings or phone calls. Sound based surveillance systems enables remote public protection by analyzing sound samples collected from the target location or the target person. Screams plays an intense role in analyzing the situation analysis for any signs of danger.

2) Speaker Identification: Speaker identification systems are used to identify a person from voice biometrics. These systems use those human voice features that differ in different individuals. Screams can be used very effectively for textindependent speaker identification.

3) Acoustic Feature Enhancement: Quite a large set of scream literature is based on the techniques that are used to improve the acoustic features enhancement of scream detection and classification. These techniques help in increasing the robustness of the detection and classification for several different kind of sound-based scream dependent systems.

\section{B. Feature Extraction}

While evaluating and characterizing the contents of an audio stream, feature extraction plays a vital role. To analyze the a scream audio stream, the first step is extracting the concerned acoustic features form the audio frames.

TABLE III. CATEGORIZATION OF AUdIO FEATURES FOR SCREAM DETECTION

\begin{tabular}{|c|c|}
\hline Category & Types \\
\hline \multirow{2}{*}{ Temporal } & Zero Crossing Rate (ZCR) \\
\hline & Short Term Energy \\
\hline \multirow{9}{*}{ Spectral } & Mel-frequency Cepstral Coefficients (MFCC) \\
\hline & Centroid \\
\hline & Roll off \\
\hline & Flux \\
\hline & Tilt \\
\hline & Spectral Entropy \\
\hline & Signal Bandwidth \\
\hline & Sub-Band Energy Ratio \\
\hline & Linear Prediction \\
\hline \multirow{4}{*}{ Prosodic } & Fundamental Frequency /Pitch \\
\hline & Intensity /Loudness \\
\hline & Duration /Rhythm \\
\hline & Log Energy \\
\hline
\end{tabular}


Table III represents different kind of acoustic features including Temporal, Spectral and Prosodic. This categorization is performed on the basis of diverse behaviour of acoustic parameters. These features can be extracted from audio signals or easy adaptability, robustness again noise and implementation.

1) Temporal: In a sound signal the amplitude fluctuation with time (the waveform signal) is represented as Temporal or time amplitude features. These acoustic features can be straightly extracted from raw sound signals for which no prior data is required. Typical temporal cases include amplitudebased features, zero-crossing rate (ZCR), and power-based features. Such features usually recommend a simple tactic to examine acoustic signals.

2) Spectral: Spectral/Cepstral features are resulted from short-term spectral features. Audio signals mostly speech and non-speech, speaker and language recognition rely on Cepstral features. The computation of cepstral is composed of three processes namely Fourier transform, inverse Fourier transform and logarithm [33]. These processes allow the identification of the purification and base frequency and of the audio signal. The different variants of Spectral features include Melfrequency Cepstral Coefficients, Spectral Centroid, Spectral Flux, Spectral Roll off, Spectral Tilt, Spectral Entropy, Signal Bandwidth, Sub-Band Energy Ratio, and Linear Prediction. Generally, the temporal features are necessarily combined with spectral features for in-depth audio analysis. Consequently, the computational complexity of spectral features is higher than that of temporal features.

3) Prosodic: In the context of human listeners, to specify information with semantic sense, prosodic/ perceptual frequency features are used. On the other hand, the prosodic features define auditory signals in terms of mathematical and physical properties. These features are ordered based on semantically eloquent characteristics of sounds. These aspects include loudness/intensity, fundamental frequency, and rhythm.

\section{Scream Classification Techniques}

Scream classification can be performed using ttraditional classification tactics. An example of such tactics includes manual classification done by human experts. The experience and skills of a good analyst make this method more reliable. Though, it is time intense and arduous in spite of the precise results. To diminish human interaction for automating the detection and classification process, two approaches are widely used and applied for scream detection and classification. These two classification approaches are supervised and unsupervised that are highlighted in Table IV along with their subtechniques. The use of semi-supervised learning algorithms is nearly non-considerable in terms of scream classification and hereby not a part of this review.
TABLE IV. MACHINE LEARNING TECHNIQUES FOR SCREAM CLASSIFICATION

\begin{tabular}{|l|l|}
\hline Category & Classification Techniques \\
\hline \multirow{5}{*}{ Supervised Learning } & K-nearest-neighbors (KNN) \\
\cline { 2 - 2 } & Neural Networks (RBF, MLP) \\
\cline { 2 - 2 } & Support Vector Machines (SVM) \\
\cline { 2 - 3 } Unsupervised & Linear Discriminants \\
\cline { 2 - 3 } Learning & Rule-based Classifiers \\
\hline \multirow{5}{*}{} & Neural Networks \\
\cline { 2 - 2 } & Hidden Markov Models (HMM) \\
\cline { 2 - 2 } & Gaussian Mixture Models (GMM) \\
\cline { 2 - 2 } & Clustering \\
\hline
\end{tabular}

1) Supervised Learning Algorithms: Supervised learning algorithms are those that aim to discover a relationship between a given input/vector and the desired output/supervisory signal. Once it analyses and figures out an association, it produces a pattern/inferred function which can be used for mapping new examples.

Supervised learning is extensively used in scream audio event detection systems. These techniques include K-nearest neighbor (k-NN), linear discriminant analysis, Bayesian networks, support vector machine, and rule-based machine algorithms. The obvious description or specification of these algorithms is to train the behavioral models with labelled data. This method holds high demand on resource consumption.

\section{a) Instance-Based or K-Nearest-Neighbors (KNN)}

The k-nearest neighbor algorithm $(\mathrm{KNN})$ is the simplest and most efficient non- parametric algorithm from the family of instance-based learning [34]. The output of this algorithm depends on whether it is used for regression or classification. $\mathrm{K}-\mathrm{NN}$ is a robust method that is proficient enough for organizing and segmenting audio streams into music, speech, environmental sounds, and silence [35]. The author in [11] used KNN for scream classification. The classification is done based on majority of neighbors. The object is allocated to the class that is in its $\mathrm{k}$ nearest neighbors where $\mathrm{k}$ is a positive integer. The value of $\mathrm{k}=1$ depicts that the object is allocated to the class of exactly that single nearest neighbor. Although $\mathrm{KNN}$ is quite easy to implement but this technique requires memory and computation complexities. To overcome this problem, [36] and many other techniques have been developed. 


\section{b) Neural Networks}

The Artificial Neural Network (ANN) is a data processing computing system which is vaguely encouraged by the biological neural networks, such as the animal or human brain process information. For audio events the Radial Basis Function (RBF) and Multi-Layer Perceptron (MLP) were applied in Artificial Neural Networks (ANNs) for supervised audio classification to decrease misclassification errors. In MLP, input datasets are mapped onto appropriate output sets. The most common use of MLP is in automatic phoneme recognition tasks [37]. A particular case [38] of feed-forward network is Radial Basis Function (RBF) which creates a linear map from the hidden space to the output space.

\section{c) Rule-Based Classifiers}

A rule-based machine learner identifies and utilize a set of relational rules that cooperatively show the knowledge captured by the system. This contrasts with the other machine learners where a singular model is commonly identified that can be applied universally on nay instance to make a prediction. A variation of this classifier is fuzzy rule-based classifier (FRBC) that is efficiently being used for numerous classification tasks. Auditory event detection in fuzzy setoriented contains the information concerning to a set of rules that classify the several characteristics of the fuzzy rule base in the training data [39]. The disadvantage of fuzzy operators is that there is no specific way to define fuzzy operators especially symbolic variables. The classification problem of non-speech human voice was solved [40] using fuzzy integral and some of the associated fuzzy measures.

\section{d) Bayesian Networks}

A Bayesian/Bayes/Belief network is a graphical model that probabilistically signifies a set of variables and their inter dependencies using a directed acyclic graph (DAG). There are the variants of the Bayesian network include: 1) serial, 2) divergent, and 3) convergent. It does fast supervised classification due to which It is appropriate for forecasting and classification tasks on complex large-scale datasets. Various multi modals [41]-[43] have been projected to resolve the glitches in acoustic and speech segmentation in movies or robot speech under noise conditions.

\section{e) Linear Discriminants}

Linear discriminant analysis (LDA) is used to find a linear combination of features that classifies two or more classes of objects or events. The resulting combination can then be used as a classifier, or for dimensionality reduction. LDA basically transfers raw data into a feature space [44] supporting a more robust classification.

\section{f) Support Vector Machines (SVM)}

Support vector machines (SVMs) are valuable machine learning method for complicated data classification problems [45]. A training set is provided to the SVM by a set called input vector. SVMs separate two types or classes by maximizing the margin between the class boundaries and the nearest ample to it.

2) Unsupervised Learning Algorithms: Unsupervised Learning algorithms are applied to infer a function or conclusions from unlabeled input data. As the data is unlabeled so its process involves finding and correlating the labels. The main objective of unsupervised learning is to examine the information and discovering similarities between the objects.

In unsupervised learning, the most common method is Cluster analysis that utilizes heuristic data for analyzing and finding hidden classes and patterns in audio data. Similarity measurement is used in clustering that is based upon metrics like Euclidean distance and probabilistic distance [46]. Some common algorithm for clustering are: 1) Gaussian Mixture Models, 2) Clustering, 3) Hidden Markov Models, and 4) Neural Networks.

\section{a) Gaussian Mixture Models (GMM)}

Gaussian mixture models (GMMs) are unsupervised classification methods. These methods are extensively used in speech/voice recognition and sensing and hence can be applied t. GMM assumes that all the data points are created from a mixture that contains several Gaussian distributions with unidentified parameters.

\section{b) Clustering}

Hierarchical clustering (HC) also called hierarchical cluster analysis is a technique of cluster analysis that is aimed at building a hierarchy of clusters by recursively merging or dividing the patterns [47], [48]. It uses two kinds of strategies. One includes constructing a hierarchy from the bottom up (agglomerative) after calculating the similarities among all duos of clusters iteratively merging the most similar pair. The other top down (Divisive) approach performs splits recursively moving down the hierarchy.

In Partitioning approaches, samples are repositioned by transferring from one cluster to the other. This method initially requires the total number of clusters that will be pre-set by the user. The well-known methods in this field include K-means and its variants [48], [49].

\section{c) Hidden Markov Models (HMM)}

A hidden Markov model (HMM) is based on unobserved or hidden variable stats. This model is a statistical Markov chain. The unobserved states are obtained based on a particular emission function that is resultant of some observable symbols [50]. The hidden Markov model can be considered to be the simplest dynamic Bayesian network. C. Chan et al. [22], M. Vacher et al. [16] used HMM for scream classification.

\section{d) Neural Networks}

Artificial neural networks (ANNs) are huge computing systems working together, consisting huge number of processors and their interconnections. The ANNs can solve reliable and efficient classification problems obtaining high tolerance and aadaptability [51]. The most commonly used neural network models for unsupervised learning algorithms are Self organizing Map (SOM) and Adaptive Resonance Theory (ART).

\section{RESULTS AND DISCUSSION}

A total of 30 research articles based on scream classification and detection are used and compared based on 
problem domains, sound features, and classification techniques. A quick analysis of the review for each case is presented below:

\section{A. Analysis of Problem Domain}

Three main problem domains for scream classification include Surveillance, Speaker Identification and Feature Enhancement. The relevant research articles are separated for each problem domain. The division of articles is hereby shown in Table V. It represents that out of 30 articles, 19 belong to individual person or public surveillance, 3 belong to the identification of the speaker and 8 discussed the methods and mechanisms to enhance and enrich the scream sound vocal experimental results. In the next step the overall percentages are calculated for these problem domains to find out which one is lagging and needs further exploration (Fig. 1).

TABLE V. Division of Problem Domains based on Literature

\begin{tabular}{|l|l|l|l|}
\hline Sr. & $\begin{array}{l}\text { Problem } \\
\text { Domain }\end{array}$ & References & Total \\
\hline 1. & Surveillance & $\begin{array}{l}\text { S. Chung et al. [9], S. Mun et al. [10], L. } \\
\text { Girin [5], Y. Li et al. [11], [12], L. H. Arnal } \\
\text { et al.[7], M. Z. Zaheer et al. [14], M. } \\
\text { Vacher et al. [16], M. Vacher et al. [17], B. } \\
\text { Uzkent et al. [20], M. Mark et al. [21], W. } \\
\text { Huang et al. [3], C. Chan et al. [22], A. } \\
\text { Fleury et al. [24], L. Gerosa et al. [2], P. C. } \\
\text { Schön et al. [27], M. Vacher et al. [28], E. } \\
\text { R. Siebert et al. [29], N. E. O. Connor et al. } \\
\text { [30] }\end{array}$ & 19 \\
\hline 2. & $\begin{array}{l}\text { Speaker } \\
\text { Identification }\end{array}$ & $\begin{array}{l}\text { J. H. L. Hansen et al.[1], M. K. Nandwana } \\
\text { et al. [15], C. Zhang et al. [25] }\end{array}$ & 3 \\
\hline 3. & $\begin{array}{l}\text { Feature } \\
\text { Enhancement }\end{array}$ & $\begin{array}{l}\text { A. Pillai et al.[8], N. Hayasaka et al. [4], J. } \\
\text { H. L. Hansen et al. [13], B. Lei et al. [18], } \\
\text { Rabaoui et al. [26], R. A. Breguet et al. } \\
\text { [31] }\end{array}$ & 8 \\
\hline
\end{tabular}

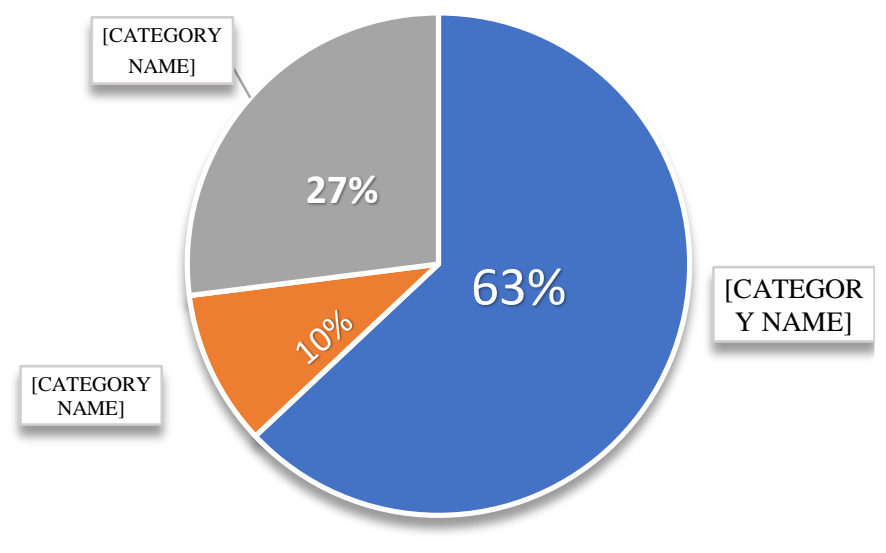

Fig. 1. Percentage usage of scream detection in various problem domains.
With the increasing rate of public crime occurrences (like on streets and transports), and danger to the precious human lives, surveillance systems based on audio analysis of screams are rapidly becoming popular. This is because the screams are usually considered and interpreted as to be the signals of survival in humans. Such systems can help majorly in medical surveys, audio scene classification, embedded transport environments like buses and trains, and 24x7 monitoring for the signs of distress in humans' daily routine.

Fig. 1 indicates that the Surveillance domain is more enriched with scream detection and classification because of the two reasons 1) Increasing number of health and safety issues and, 2) Screams are a sign of danger.

\section{B. Analysis of Scream Sound Features}

It is computationally expensive to utilize all the sound features for scream classification, so it is a common practice to mix-up one or two type of features together to achieve the best results in conjunction with classification techniques.

TABLE VI. TAXONOMY OF SCREAM FEATURE TYPES

\begin{tabular}{|c|c|c|}
\hline Sr. & Feature Type & References \\
\hline 1. & Spectral (S) & $\begin{array}{l}\text { L. Girin [5], S. Chung et al. [9], S. Mun et al. [10], } \\
\text { Y. Li et al. [11], A. Sharma et al. [12], M. K. } \\
\text { Nandwana et al. [15], M. Vacher et al. [16], B. Lei et } \\
\text { al. [18], A. Fleury et al. [24], A. Rabaoui et al. [26], } \\
\text { P. C. Schön et al. [27], R. A. Breguet et al. [31] }\end{array}$ \\
\hline 2. & Prosodic $(\mathrm{P})$ & K. Kato [19] \\
\hline 3. & $\begin{array}{l}\text { Temporal, } \\
\text { Spectral (TP) }\end{array}$ & $\begin{array}{l}\text { J. H. L. Hansen et al.[1], A. Pillai et al.[8], M. } \\
\text { Vacher et al. [17], M. Vacher et al. [28], E. R. } \\
\text { Siebert et al. [29], N. E. O. Connor et al. [30] }\end{array}$ \\
\hline 4. & $\begin{array}{l}\text { Spectral, } \\
\text { Prosodic (SP) }\end{array}$ & $\begin{array}{l}\text { W. Huang et al. [3], N. Hayasaka et al. [4], M. Z. } \\
\text { Zaheer et al. [14], B. Uzkent et al. [20], W. Liao et } \\
\text { al. [23], C. Zhang et al. [25] }\end{array}$ \\
\hline 5. & $\begin{array}{l}\text { Temporal, } \\
\text { Spectral, } \\
\text { Prosodic } \\
\text { (TSP) }\end{array}$ & $\begin{array}{l}\text { L. Gerosa et al. [2], L. H. Arnal et al.[7], J. H. L. } \\
\text { Hansen et al. [13], M. Mark et al. [21], C. Chan et al. } \\
\text { [22] }\end{array}$ \\
\hline
\end{tabular}

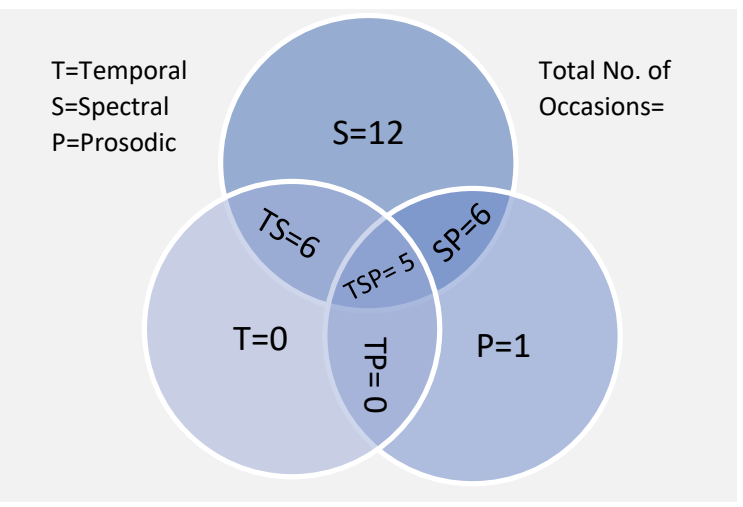

Fig. 2. Basic Venn diagram for the use of sound parameter on several occasions. 


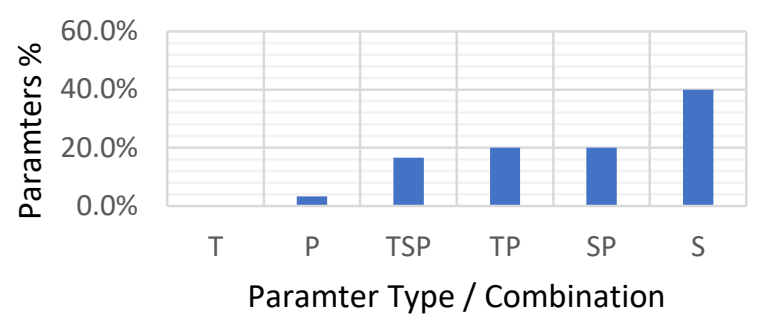

Fig. 3. Percentage usage of individual and combined parameter.

While exploring the sound features it can be observed that some of the articles are using the combined feature approach. Following this, a taxonomy has been developed (described in Table VI). The temporal features cannot be effectively used separately so no article has independently used these features but in combination with other types.

Spectral and Prosodic features are used independently as well as in combination. Table VI describes all of the articles under consideration and the type of sound features they have used or recommended for scream classification. The results of this step are shown in Fig. 2 and 3.

In Fig. 2, $\mathrm{S}=$ =Spectral, $\mathrm{P}=$ =Prosodic, $\mathrm{T}=$ Temporal, $\mathrm{TS}=$ Temporal and Spectral, $\mathrm{SP}=$ Spectral and Prosodic, $\mathrm{TP}=$ Temporal and Prosodic and TSP $=$ Temporal, Spectral and Prosodic. It also shows that the most commonly used sound features are spectral. Out of 30 researches, 12 used spectral features independently. The second-best features are the combination of either TS or SP. While no one recommended T or TP.
The results are presented by calculating the percentages for each type or combination. The percentage evaluation is shown in Fig. 3 which clearly expresses that the spectral parameters are the most recommended ones to achieve the best scream classification with $40 \%$ of usability.

Further we see that there are further many forms of each category of scream sound feature. Table VII describes all the considered scream articles with the type of sound feature they have used in detail.

In the last step, it has been concluded that spectral features are highly recommended in literature for scream classification. The basic purpose of this step is to figure out that out of many forms of Spectral features which one shows the best performance out of all.

Fourier transform is used to convert time-domain signal into frequency domain for obtaining spectral features. These features are quite helpful in identifying the notes, pitch, rhythms and melody.

The results of this step are shown in Fig. 4. It can be clearly observed that Mel-frequency Cepstral Coefficients are the most used and highly recommended sound feature for scream classification. It can either be used individually or in combination with other sound features. MFCC are extensively applied in voice recognition because of the reason that these features are very similar to human listening. In more complicated and complex signals such as speech or music where the signal changes its properties over time, it is evidently more meaningful to refer to the altering frequency content over a smaller time interval than an infinite time interval.

TABLE VII. AUdio FEATURE CATEGORIZATION FOR SCREAM DETECTION AND ClaSSiFiCATION

\begin{tabular}{|c|c|c|}
\hline Category & Types & References \\
\hline \multirow[b]{2}{*}{ Temporal } & Zero Crossing Rate (ZCR) & A. Pillai et al.[8], M. Vacher et al. [17], M. Vacher et al. [28], C. Chan et al. [22], L. Gerosa et al. [2] \\
\hline & Short Term Energy & $\begin{array}{l}\text { M. Mark et al. [21], A. Pillai et al.[8], J. H. L. Hansen et al.[1], L. H. Arnal et al.[7], J. H. L. Hansen et al. [13], } \\
\text { E. R. Siebert et al. [29], N. E. O. Connor et al. [30] }\end{array}$ \\
\hline \multirow{9}{*}{ Spectral } & $\begin{array}{l}\text { Mel-frequency Cepstral } \\
\text { Coefficients } \\
\text { (MFCC) }\end{array}$ & $\begin{array}{l}\text { M. Mark et al. [21], J. H. L. Hansen et al.[1], N. Hayasaka et al. [4], L. H. Arnal et al.[7], M. Vacher et al. [28], } \\
\text { S. Chung et al. [9], L. Gerosa et al. [2], S. Mun et al. [10], L. Girin [5], Y. Li et al. [11], A. Sharma et al. [12], J. } \\
\text { H. L. Hansen et al. [13], M. Vacher et al. [16], B. Lei et al. [18], W. Huang et al. [3], A. Fleury et al. [24], A. } \\
\text { Rabaoui et al. [26], M. K. Nandwana et al. [15], B. Uzkent et al. [20], W. Liao et al. [23], C. Zhang et al. [25] }\end{array}$ \\
\hline & Spectral Centroid & $\begin{array}{l}\text { M. Mark et al. [21], A. Pillai et al.[8], M. Vacher et al. [17], M. Vacher et al. [28], L. Gerosa et al. [2],, R. A. } \\
\text { Breguet et al. [31], W. Liao et al. [23], }\end{array}$ \\
\hline & Spectral Roll off & A. Pillai et al.[8], M. Vacher et al. [17], M. Vacher et al. [28], L. Gerosa et al. [2], W. Liao et al. [23] \\
\hline & Spectral Flux & E. R. Siebert et al. [29], L. Gerosa et al. [2],, M. Z. Zaheer et al. [14], R. A. Breguet et al. [31] \\
\hline & Spectral Tilt & L. Gerosa et al. [2], R. A. Breguet et al. [31], C. Zhang et al. [25] \\
\hline & Spectral Entropy & M. Mark et al. [21], A. Pillai et al.[8] , N. Hayasaka et al. [4], W. Liao et al. [23] \\
\hline & Signal Bandwidth & M. Mark et al. [21], W. Liao et al. [23] \\
\hline & Sub-Band Energy Ratio & J. H. L. Hansen et al.[1], C. Chan et al. [22], M. Z. Zaheer et al. [14], C. Zhang et al. [25] \\
\hline & Linear Prediction & P. C. Schön et al. [27], N. E. O. Connor et al. [30] \\
\hline \multirow{4}{*}{ Prosodic } & $\begin{array}{l}\text { Pitch/Fundamental } \\
\text { Frequency }\end{array}$ & $\begin{array}{l}\text { M. Mark et al. [21], L. H. Arnal et al.[7], C. Chan et al. [22], L. Gerosa et al. [2], J. H. L. Hansen et al. [13], M. } \\
\text { Z. Zaheer et al. [14], K. Kato [19], B. Uzkent et al. [20], W. Liao et al. [23] }\end{array}$ \\
\hline & Loudness/Intensity & L. Gerosa et al. [2], K. Kato [19], C. Zhang et al. [25] \\
\hline & Rhythm/Duration & C. Chan et al. [22], K. Kato [19], C. Zhang et al. [25] \\
\hline & Log Energy & N. Hayasaka et al. [4], W. Huang et al. [3] \\
\hline
\end{tabular}




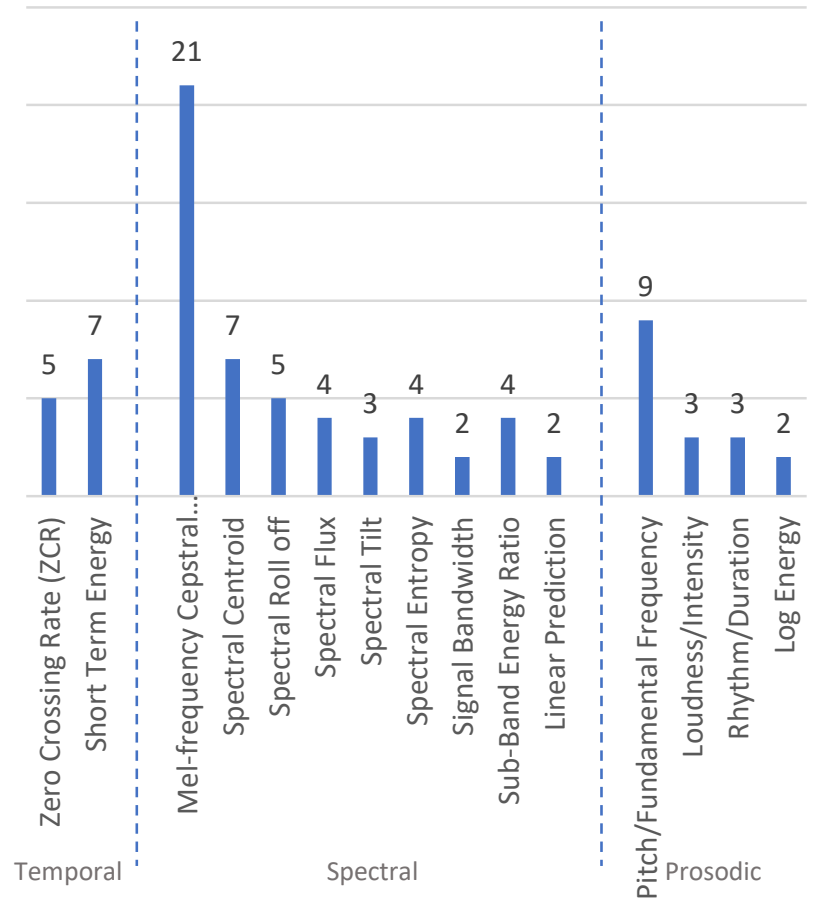

Fig. 4. Division of articles based on sound features.

\section{Analysis of Classification Techniques}

There are two clear divisions of sound event detection approaches: supervised, unsupervised or combination. These approaches are studied perceptibly however still suffer from a scarcity of additional thorough and complete analysis on classification approaches, primarily in scream signal classification. This review documents the scream classification with two subclasses in conjunction with a close review of every class.

This taxonomy has been shown in Table VIII. The referenced articles in each category are carefully observed and assigned to the relevant class. Some of the techniques are using supervised and unsupervised approach independently while the others are using a combination of both approaches (separately). This table is not for comparison as the datasets and the sound features are used differently. It is just providing a review of the current illustrative approaches.

A more precise view is presented in Fig. 5, where 11 researches used supervised, 13 used un-supervised and 4 used combined scream classification approaches. Furthermore, the generic analytical view of classification approach is shown in Fig. 6, where the percentage calculations are performed in each case. It can clearly be seen that the un-supervised approaches have been more successfully been applied than other approaches in the last 18 years for scream detection and classification. For this purpose, the supervised and unsupervised scream classification techniques are further explained and analyzed in the next section.

1) Supervised Learning Algorithms: Supervised learning algorithms are categorized as K-nearest-neighbors (KNN) or instance-based, neural networks, rule-based Classifiers, linear discriminant, Bayesian networks, and support vector machines (SVM).

The primary purpose of this review is to present supervised learning approaches based on scream classification. The future researchers can find out the ways to explore the automated acoustic environments and systems. The most recent experimental research works related to screams classifications and detection are summarized in Table IX. It presents the latest methods for undertaking scream classification and detection issues based on supervised learning methods.

Accuracies of classifiers are sstatistically compared and calculated by finding out the total no. of researches along with their classification results. By finding the individual accuracy of each supervised learning classification technique mentioned in the literature, average accuracies have been calculated to find out which techniques is providing the best results.

Fig. 7 shows the percentage accuracies of each technique along with the authors and references independently. $\mathrm{N}$. Hayasaka et al. [4] is leading while using SVMs with accuracy rate of $94.6 \%$.

TABLE VIII. SCREAM ClassificATION TECHNIQUES

\begin{tabular}{|l|l|l|}
\hline Sr. & Feature Type & References \\
\hline 1. & Supervised & $\begin{array}{l}\text { W. Huang et al. [3], L. Girin [5], L. H. Arnal et al.[7], A. Pillai et al.[8], A. Sharma et al. [12], B. Lei et al. [18], B. Uzkent et } \\
\text { al. [20], M. Mark et al. [21], W. Liao et al. [23], A. Rabaoui et al. [26], P. C. Schön et al. [27], }\end{array}$ \\
\hline 2. & $\begin{array}{l}\text { Un- Supervised } \\
\text { J. H. L. Hansen et al.[1], L. Gerosa et al. [2], S. Mun et al. [10], J. H. L. Hansen et al. [13], M. Z. Zaheer et al. [14], M. K. } \\
\text { Nandwana et al. [15], M. Vacher et al. [16], M. Vacher et al. [17], C. Chan et al. [22], A. Fleury et al. [24], C. Zhang et al. [25], } \\
\text { N. E. O. Connor et al. [30], R. A. Breguet et al. [31] }\end{array}$ \\
\hline 3. & Both & N. Hayasaka et al. [4], S. Chung et al. [9], Y. Li et al. [11], M. Vacher et al. [28]
\end{tabular}




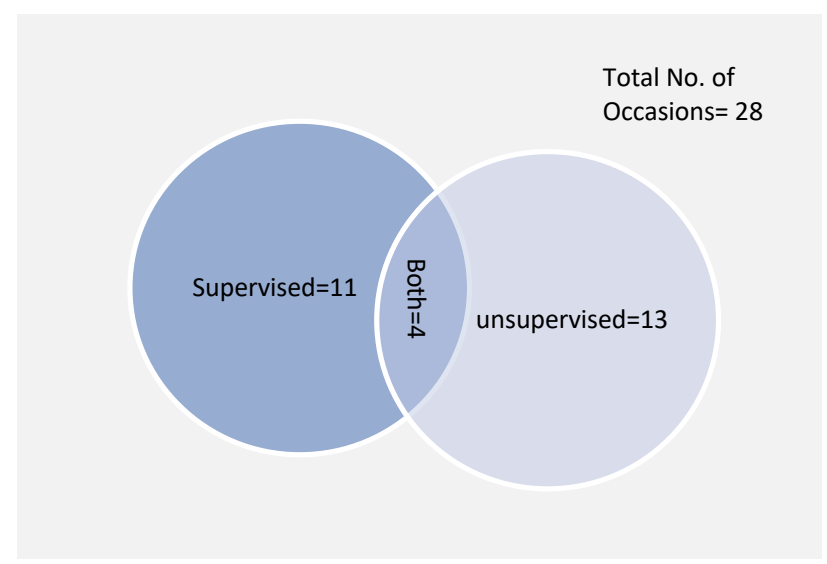

Fig. 5. Representation of scream classification techniques.

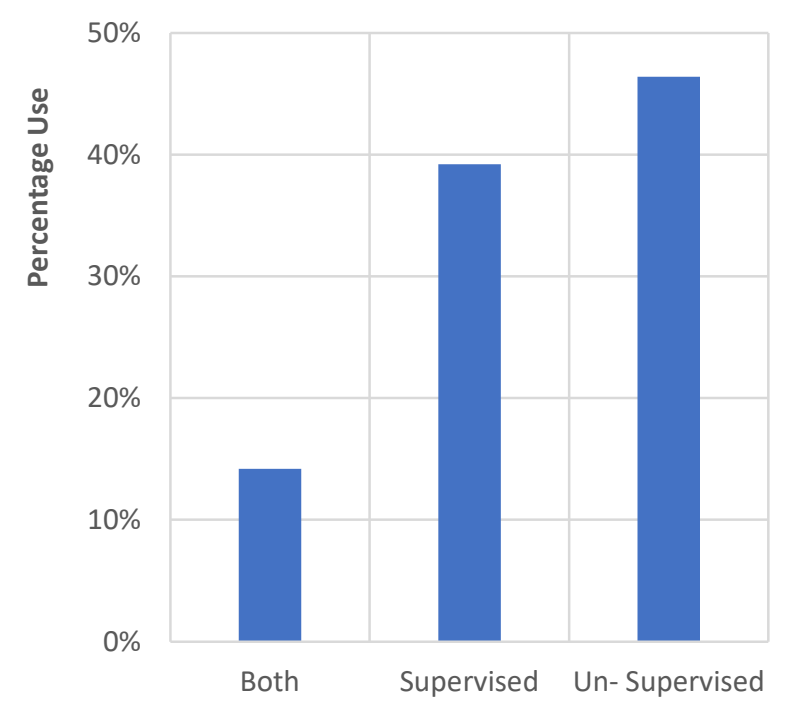

Machine Learning Techniques

Fig. 6. Percentage usage of machine learning techniques.

Fig. 8 shows the average accuracies of all supervised scream classification techniques. It can be clearly observed that Linear discriminants are producing the highest accuracy rate of $96.1 \%$ and after that the KNN with accuracy rate of $94 \%$.

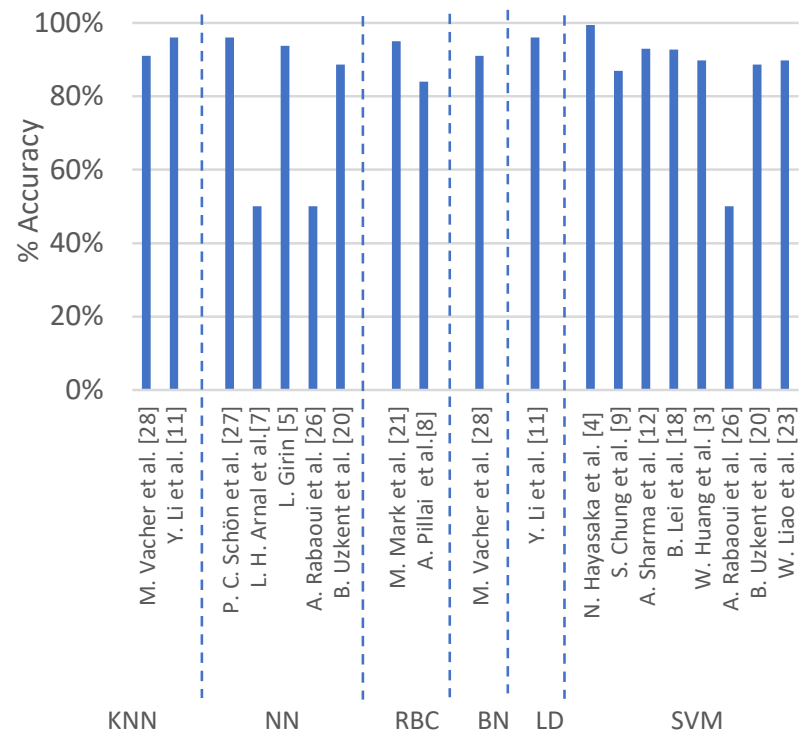

Fig. 7. Accuracies of supervised scream classifiers.

2) Unsupervised Learning Algorithms: Unsupervised learning algorithms comprehend a major learning paradigm and have drawn considerable attention in past few decades, as shown by the growing range of research publications in this field. The unsupervised methods for scream detection and classification are classified into four classes: Clustering, GMM, HMM and NN.

Table $\mathrm{X}$ lists the most significant research works and their average accuracies dealing with scream detection and classification problems associated with unsupervised approaches to present some solutions to the problems restraining the performance of scream classification systems for situation understanding.

Fig. 9 shows the percentage accuracies of each technique along with the authors and references independently. M.Z. Zaheer et al. [14] achieved 100\% scream detection accuracy with GMM technique. Another classification technique used by N. Hayasaka et al. [4] achieved an accuracy rate of $99 \%$ again with GMM.

TABLE IX. SCREAM ClASSIFICATION TECHNIQUES

\begin{tabular}{|c|c|c|c|c|}
\hline Category & Classification Techniques & References & Total Articles & $\begin{array}{l}\text { Average } \\
\text { Accuracies }\end{array}$ \\
\hline \multirow{6}{*}{$\begin{array}{l}\text { Supervised } \\
\text { Learning }\end{array}$} & $\begin{array}{l}\text { Instance-based or K-nearest- } \\
\text { neighbors }(\mathrm{KNN})\end{array}$ & M. Vacher et al. [28], Y. Li et al. [11] & 2 & $94 \%$ \\
\hline & Neural Networks (RBF, MLP) & $\begin{array}{l}\text { P. C. Schön et al. [27], L. H. Arnal et al.[7], L. Girin [5], A. Rabaoui et } \\
\text { al. [26], B. Uzkent et al. [20] }\end{array}$ & 5 & $76 \%$ \\
\hline & Rule-based Classifiers & M. Mark et al. [21], A. Pillai et al.[8] & 2 & $90 \%$ \\
\hline & Bayesian Networks & M. Vacher et al. [28] & 1 & $91 \%$ \\
\hline & Linear Discriminants & Y. Li et al. [11] & 1 & $96.1 \%$ \\
\hline & Support Vector Machines (SVM) & $\begin{array}{l}\text { N. Hayasaka et al. [4], S. Chung et al. [9], A. Sharma et al. [12], B. Lei et } \\
\text { al. [18], W. Huang et al. [3], A. Rabaoui et al. [26], B. Uzkent et al. [20], } \\
\text { W. Liao et al. [23] }\end{array}$ & 8 & $86.3 \%$ \\
\hline
\end{tabular}


TABLE X. UNSUPERVISED LEARNING TECHNIQUES FOR SCREAM CLASSIFICATION

\begin{tabular}{|c|c|c|c|c|}
\hline Category & Classification Techniques & References & $\begin{array}{l}\text { Total } \\
\text { Articles }\end{array}$ & $\begin{array}{l}\text { Average } \\
\text { Accuracies }\end{array}$ \\
\hline \multirow{4}{*}{$\begin{array}{l}\text { Unsupervised } \\
\text { Learning } \\
\text { Algorithms }\end{array}$} & $\begin{array}{l}\text { Hierarchical and } \\
\text { Partition Clustering }\end{array}$ & N. E. O. Connor et al.[30], M. K. Nandwana et al. [15], & 2 & $76 \%$ \\
\hline & Gaussian Mixture Models (GMM) & $\begin{array}{l}\text { J. H. L. Hansen et al.[1], } \\
\text { N. Hayasaka et al. [4], } \\
\text { M. Vacher et al. [17], M. Vacher et al. [28], S.Chung et al. [9], L. } \\
\text { Gerosa et al. [2], S. Mun et al. [10], Y. Li et al. [11], M. Z. Zaheer et } \\
\text { al. [14], M. Vacher et al. [16], A. Fleury et al. [24], R. A. Breguet et } \\
\text { al. [31], C. Zhang et al. [25] }\end{array}$ & 13 & $86 \%$ \\
\hline & Hidden Markov Models (HMM) & $\begin{array}{l}\text { C. Chan et al. [22], M. Vacher et al. [16], A. Fleury et al. [24], R. A. } \\
\text { Breguet et al. [31] }\end{array}$ & 4 & $75 \%$ \\
\hline & $\begin{array}{l}\text { Neural networks (Self-organizing } \\
\text { map) }\end{array}$ & S. Mun et al. [10], J. H. L. Hansen et al. [13] & 2 & $83 \%$ \\
\hline
\end{tabular}

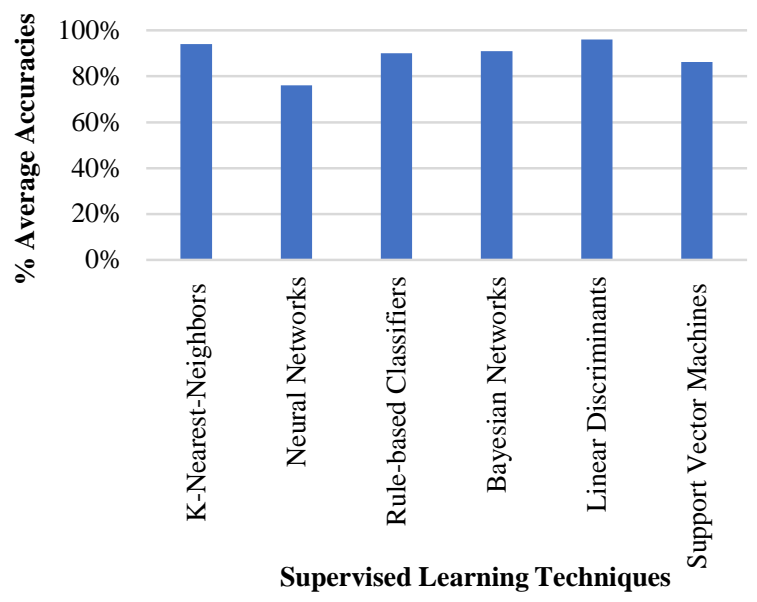

Fig. 8. Average accuracies of supervised scream classifiers.

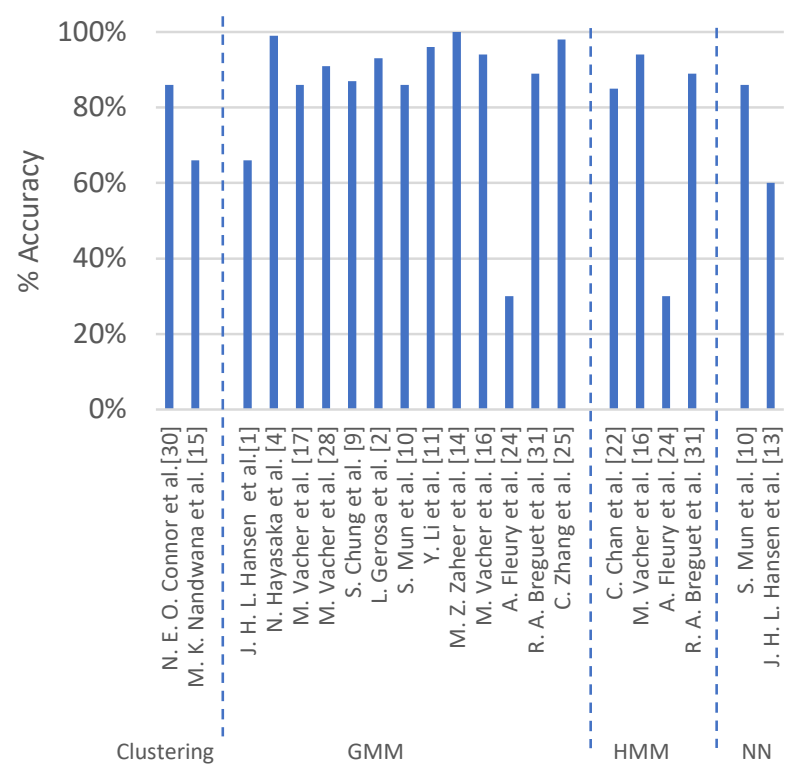

Fig. 9. Accuracies of unsupervised scream classifiers.
$100 \%$

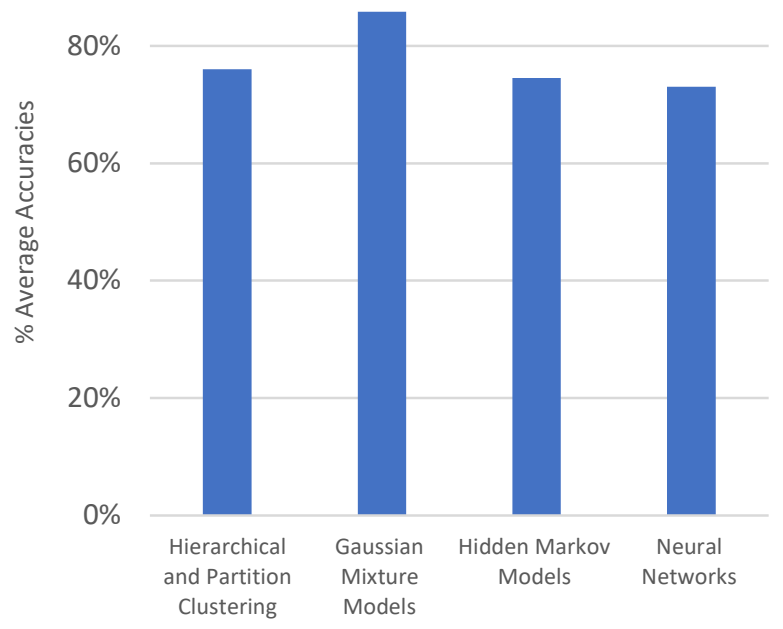

Un-Supervied Learning Techniques

Fig. 10. Average accuracies of un-supervised scream classifiers.

The overall average accuracies of the four un-supervised scream classifiers are calculated and plotted in Fig. 10. It can clearly be observed that GMMs are producing the best results with an average classification accuracy rate of $86 \%$.

\section{a) Combining Results}

The results of overall review are converged in Table XI. It shows all of the research articles of last 18 years (from 20002018) based on the specified sound parameters and classification techniques. The accuracy percentage and the effective error rate (ERR) for each article is also mentioned. Fig. 11 plots theses results for scream detection and scream sound classification.

Maximum percentage accuracies are used. Some of the researchers have done descriptive studies so that if the numerical results are not provided then these are supposed to be $50 \%$ accurate to show that the results are encouraging. 
TABLE XI. BRIEF REVIEW OF SCREAM LITERATURE

\begin{tabular}{|c|c|c|c|c|c|c|c|c|c|c|c|c|c|c|}
\hline \multirow[b]{2}{*}{$\#$} & \multirow[b]{2}{*}{ Name/ Ref. } & \multirow[b]{2}{*}{ Year } & \multicolumn{3}{|c|}{ Problem Domain } & \multicolumn{3}{|c|}{$\begin{array}{l}\text { Sound } \\
\text { Parameters }\end{array}$} & \multicolumn{2}{|c|}{$\begin{array}{l}\text { Classification } \\
\text { Technique/es }\end{array}$} & \multicolumn{2}{|c|}{$\begin{array}{l}\text { Detection/ } \\
\text { Classification }\end{array}$} & \multicolumn{2}{|l|}{ Results } \\
\hline & & & 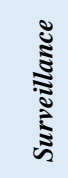 & 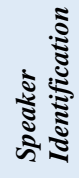 & 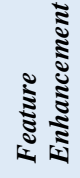 & 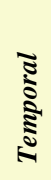 & 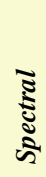 & 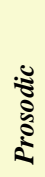 & 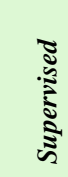 & 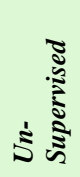 & 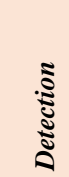 & 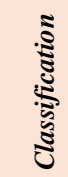 & $\begin{array}{l}\% \\
\text { Accuracy }\end{array}$ & $\begin{array}{l}\% \\
\text { EER }\end{array}$ \\
\hline 1. & A. Pillai et al.[8] & 2018 & & & $\checkmark$ & $\checkmark$ & $\checkmark$ & & $\checkmark$ & & $\checkmark$ & & $84 \%$. & $16 \%$ \\
\hline 2. & J. H. L. Hansen et al.[1] & 2017 & & $\checkmark$ & & $\checkmark$ & $\checkmark$ & & & $\checkmark$ & $\checkmark$ & & $66.67 \%$ & $33.33 \%$ \\
\hline 3. & N. Hayasaka et al. [4] & 2017 & & & $\checkmark$ & & $\checkmark$ & $\checkmark$ & $\checkmark$ & $\checkmark$ & $\checkmark$ & & $99.45 \%$ & $0.55 \%$ \\
\hline 4. & S. Chung et al. [9] & 2017 & $\checkmark$ & & & & $\checkmark$ & & $\checkmark$ & $\checkmark$ & $\checkmark$ & & $87.035 \%$ & $12.965 \%$ \\
\hline 5. & S. Mun et al. [10] & 2017 & $\checkmark$ & & & & $\checkmark$ & & & $\checkmark$ & $\checkmark$ & & $86.3 \%$. & $13.7 \%$ \\
\hline 6. & L. Girin [5] & 2016 & $\checkmark$ & & & & $\checkmark$ & & $\checkmark$ & & $\checkmark$ & & $93.8 \%$ & $6.2 \%$ \\
\hline 7. & Y. Li et al. [11] & 2016 & $\checkmark$ & & & & $\checkmark$ & & $\checkmark$ & $\checkmark$ & $\checkmark$ & & $96.1 \%$ & $3.9 \%$ \\
\hline 8. & A. Sharma et al. [12] & 2016 & $\checkmark$ & & & & $\checkmark$ & & $\checkmark$ & & $\checkmark$ & & $93.16 \%$ & $6.84 \%$ \\
\hline 9. & L. H. Arnal et al.[7] & 2015 & $\checkmark$ & & & $\checkmark$ & $\checkmark$ & $\checkmark$ & $\checkmark$ & & $\checkmark$ & & $50 \%$ & $50 \%$ \\
\hline 10. & J. H. L. Hansen et al. [13] & 2015 & & & $\checkmark$ & $\checkmark$ & $\checkmark$ & $\checkmark$ & $\checkmark$ & & $\checkmark$ & & $60 \%$ & $40 \%$ \\
\hline 11. & M. Z. Zaheer et al. [14] & 2015 & $\checkmark$ & & & & $\checkmark$ & $\checkmark$ & & $\checkmark$ & $\checkmark$ & & $100 \%$ & $0 \%$ \\
\hline 12. & M. K. Nandwana et al. [15] & 2014 & & $\checkmark$ & & & $\checkmark$ & & & $\checkmark$ & $\checkmark$ & & $66.67 \%$ & $33.33 \%$ \\
\hline 13. & M. Vacher et al. [16] & 2014 & $\checkmark$ & & & & $\checkmark$ & & & $\checkmark$ & $\checkmark$ & & $94 \%$ & $06 \%$ \\
\hline 14. & M. Vacher et al. [17] & 2014 & $\checkmark$ & & & $\checkmark$ & $\checkmark$ & & & $\checkmark$ & $\checkmark$ & & $86.46 \%$ & $13.54 \%$ \\
\hline 15. & B. Lei et al. [18] & 2014 & & & $\checkmark$ & & $\checkmark$ & & $\checkmark$ & & $\checkmark$ & & $92.76 \%$ & $7.24 \%$ \\
\hline 16. & K. Kato [19] & 2013 & & & $\checkmark$ & & & $\checkmark$ & N/A & N/A & $\checkmark$ & & $50 \%$ & $50 \%$ \\
\hline 17. & B. Uzkent et al. [20] & 2012 & $\checkmark$ & & & & $\checkmark$ & $\checkmark$ & $\checkmark$ & & $\checkmark$ & & $88.7 \%$ & $11.3 \%$ \\
\hline 18. & M. Mark et al. [21] & 2012 & $\checkmark$ & & & $\checkmark$ & $\checkmark$ & $\checkmark$ & $\checkmark$ & & $\checkmark$ & & $95 \%$ & $05 \%$ \\
\hline 19. & W. Huang et al. [3] & 2010 & $\checkmark$ & & & & $\checkmark$ & $\checkmark$ & $\checkmark$ & & $\checkmark$ & & $89.815 \%$ & $10.185 \%$ \\
\hline 20. & C. Chan et al. [22] & 2010 & $\checkmark$ & & & $\checkmark$ & $\checkmark$ & $\checkmark$ & & $\checkmark$ & $\checkmark$ & & $85.75 \%$ & $14.25 \%$ \\
\hline 21. & W. Liao et al. [23] & 2009 & & & $\checkmark$ & & $\checkmark$ & $\checkmark$ & $\checkmark$ & & $\checkmark$ & & $89.81 \%$ & $10.19 \%$ \\
\hline 22. & A. Fleury et al. [24] & 2008 & $\checkmark$ & & & & $\checkmark$ & & & $\checkmark$ & $\checkmark$ & & $30.43 \%$ & $69.57 \%$ \\
\hline 23. & L. Gerosa et al. [2] & 2007 & $\checkmark$ & & & $\checkmark$ & $\checkmark$ & $\checkmark$ & & $\checkmark$ & $\checkmark$ & & $93 \%$, & $07 \%$ \\
\hline 24. & C. Zhang et al. [25] & 2007 & & $\checkmark$ & & & $\checkmark$ & $\checkmark$ & & $\checkmark$ & $\checkmark$ & & $98.5 \%$ & $1.5 \%$ \\
\hline 25. & A. Rabaoui et al. [26] & 2007 & & & $\checkmark$ & & $\checkmark$ & & $\checkmark$ & & $\checkmark$ & & $50 \%$ & $50 \%$ \\
\hline 26. & P. C. Schön et al. [27] & 2004 & $\checkmark$ & & & & $\checkmark$ & & $\checkmark$ & & $\checkmark$ & $\checkmark$ & $96 \%$ & $04 \%$ \\
\hline 27. & M. Vacher et al. [28] & 2004 & $\checkmark$ & & & $\checkmark$ & $\checkmark$ & & $\checkmark$ & $\checkmark$ & $\checkmark$ & & $91 \%$ & $09 \%$ \\
\hline 28. & E. R. Siebert et al. [29] & 2003 & $\checkmark$ & & & $\checkmark$ & $\checkmark$ & & & N/A & $\checkmark$ & & $50 \%$ & $50 \%$ \\
\hline $29 .$. & N. E. O. Connor et al. [30] & 2002 & $\checkmark$ & & & $\checkmark$ & $\checkmark$ & & & $\checkmark$ & $\checkmark$ & & $86.67 \%$ & $13.33 \%$ \\
\hline 30. & R. A. Breguet et al. [31] & 2000 & & & $\checkmark$ & & $\checkmark$ & & & $\checkmark$ & $\checkmark$ & & $89 \%$ & $11 \%$ \\
\hline
\end{tabular}




\section{\% Accuracy $\square$ EER \%}

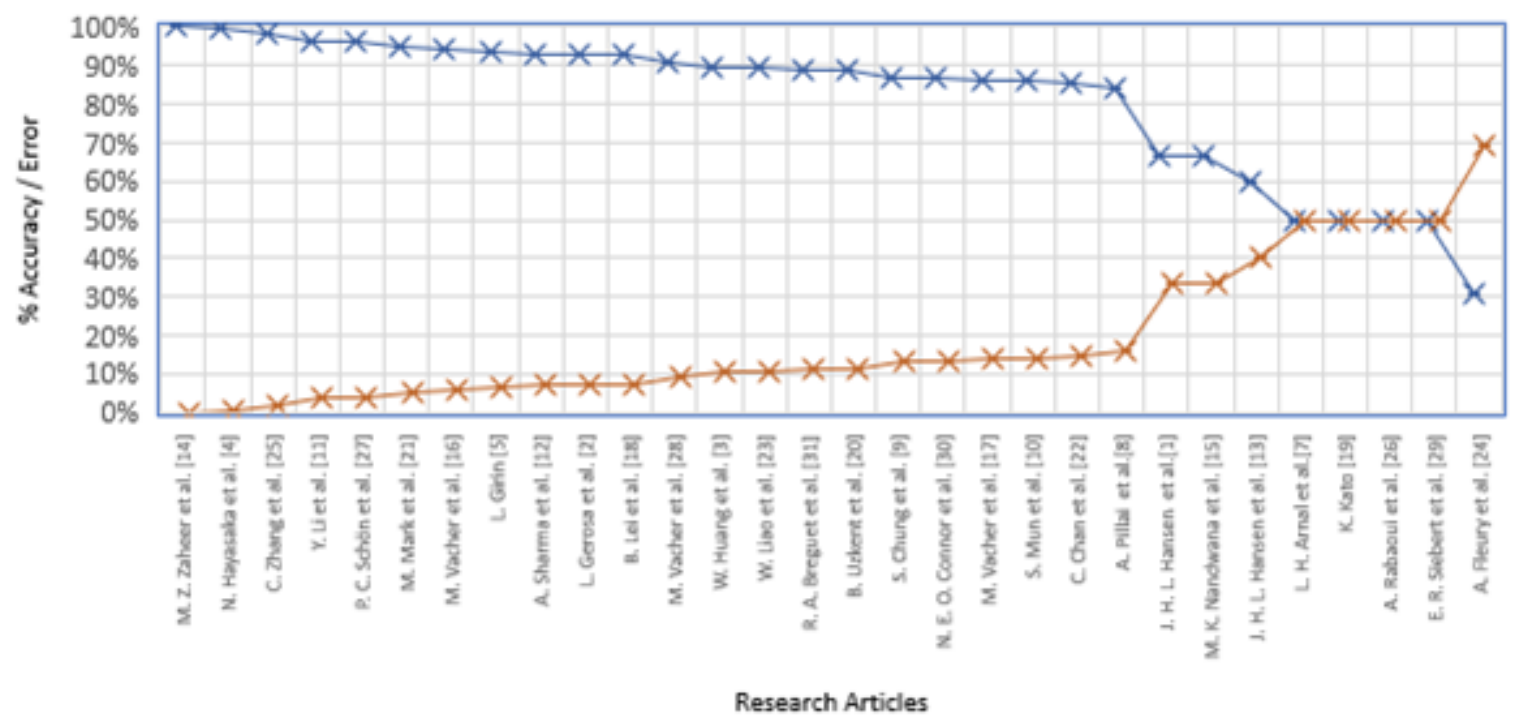

Fig. 11. Accuracy and EER for scream detection and classification.

It can be clearly observed that only a single research conducted by P. C. Schön et al. [27] in 2004 has focused on scream detection as well as classification. But this research is based on chimpanzee screams. The authors have figured out the ways in which the chimpanzees can be understood and what different kind of meanings can be driven from their screams. Two of the researches i.e. K. Kato [19] and E. R. Siebert et al. [29], have not used machine learning techniques instead they have developed their own for scream detection and classification.

So, there is a clear and a wide scope for scream classification non-understanding the situations in which they occur and to support the embedded sound-based systems especially surveillance systems to make the humans and animals out of danger.

\section{CONCLUSION}

A thorough analysis is presented on researchers' attempts related to scream detection and classification techniques. An in-depth taxonomy of scream detection and classification systems was presented in this review. The concerning efforts are expected to maximize scream signal detection and classification accuracy and understanding the surrounding situation of a speaker. The focus of this review is on machine learning and classification methods as well as essential sound parameters for scream-based audio embedded systems.

Although the best combination that can be concluded is that for the case of scream classification, unsupervised learning technique i.e. GMM can be applied using spectral sound features necessarily including MFCC in the field of surveillance. Because in surveillance scream detection and sound classification has been implemented in remarkably high percentage, so there are chances that the surveillance systems based on scream detection and classification, are causing a higher risk to humanity. But these results are concluded on the information and statistics that is based on different kind of data sets using various combinations of sound parameters and classification techniques. The results may vary based on the datasets used and the background noise level.

In future, this review can be beneficial for the researchers to conduct a mechanism for scream classification and to understand the best possible alternatives in terms of classification techniques and sound parameters. A system can be developed using the concluded research to find out the differences in different classes of screams like joy, fear, sadness, etc. and to find out that how such kind of research can be helpful for understanding the surroundings of a speaker.

\section{REFERENCES}

[1] J. H. L. Hansen, M. K. Nandwana, and N. Shokouhi, "Analysis of human scream and its impact on text-independent," vol. 2957, 2017.

[2] L. Gerosa, M. Tagliasacchi, F. Antonacci, and I. Politecnico, "Scream and Gunshot Detection and Localization for Audio-Surveillance Systems," 2007.

[3] W. Huang, T. K. Chiew, H. Li, T. S. Kok, and J. Biswas, "Scream Detection for Home Applications," pp. 15-18, 2010.

[4] N. Hayasaka, A. Kawamura, and N. Sasaoka, "Noise-robust scream detection using band-limited spectral entropy," AEUE - Int. J. Electron. Commun., vol. 76, pp. 117-124, 2017.

[5] L. Girin, "Deep Neural Networks For Automatic Detection Of Screams And Shouted Speech In Subway Trains Ifsttar, COSYS, LEOST, Villeneuve d' Ascq INRIA Grenoble Rh^," pp. 6460-6464, 2016.

[6] B. Lei and M. Mak, "Robust scream sound detection via sound event partitioning," 2015.

[7] L. H. Arnal et al., "Human Screams Occupy a Privileged Niche in the Communication Soundscape Report Human Screams Occupy a Privileged Niche in the Communication Soundscape," Curr. Biol., pp. 16, 2015.

[8] A. Pillai and P. Kaushik, "AC : An Audio Classi fi er to Classify Violent Extensive Audios," 2018.

[9] S. Chung and Y. Chung, "Scream sound detection based on SVM and GMM," no. 1, pp. 1-4, 2017.

[10] S. Mun, S. Shon, W. Kim, D. K. Han, and H. Ko, "Deep Neural Network Based Learning and Transferring Mid-Level Audio Features 
for Acoustic Scene Classification,”pp. 796-800, 2017.

[11] Y. Li and G. Liu, "Sound Classification Based On Spectrogram," no. 2, 2016.

[12] A. Sharma and S. Kaul, "Two-Stage supervised learning-based method to detect screams and cries in urban environments," IEEE/ACM Trans. Audio Speech Lang. Process., vol. 24, no. 2, pp. 290-299, 2016.

[13] J. H. L. H. Mahesh Kumar Nandwana, Ali Ziaei, "Robust Unsupervised Detection Of Human Screams In Noisy Acoustic Environments," pp. 161-165, 2015.

[14] M. Z. Zaheer, J. Y. Kim, H. G. Kim, and S. Y. Na, “A preliminary study on deep-learning based screaming sound detection," 2015 5th Int. Conf. IT Converg. Secur. ICITCS 2015 - Proc., 2015.

[15] M. K. Nandwana, J. H. L. Hansen, E. Jonsson, and C. Science, "Analysis and Identification of Human Scream: Implications for Speaker Recognition," no. September, pp. 2253-2257, 2014.

[16] M. Vacher and M. Vacher, "Sound Classification in a Smart Room Environment : an Approach using GMM and HMM Methods," 2014.

[17] M. Vacher et al., "Sound Detection and Classification for Medical Telesurvey," 2014.

[18] B. Lei, "Sound-Event Partitioning and Feature Normalization for Robust Sound-Event Detection," no. August, pp. 389-394, 2014.

[19] K. Kato and A. Ito, "Acoustic features and auditory impressions of death growl and screaming voice," Proc. - 2013 9th Int. Conf. Intell. Inf. Hiding Multimed. Signal Process. IIH-MSP 2013, pp. 460-463, 2013.

[20] B. Uzkent, B. D. Barkana, and H. Cevikalp, "Non-speech environmental sound classification using SVMs with a new set of features," no. January 2015, 2012.

[21] M. Mak and S.-Y. Kung, "Low-Power Svm Classifiers For Sound Event Classification On Mobile Devices," pp. 1985-1988, 2012.

[22] C. Chan and E. W. M. Yu, "An Abnormal Sound Detection and Classification System for Surveillance Applications," pp. 1851-1855, 2010.

[23] W. Liao and Y. Lin, "Classification of Non-Speech Human Sounds: Feature Selection and Snoring Sound Analysis," no. October, pp. 2695$2700,2009$.

[24] A. Fleury, N. Noury, M. Vacher, H. Glasson, and J.-F. Seri, "Sound and speech detection and classification in a Health Smart Home," 2008 30th Annu. Int. Conf. IEEE Eng. Med. Biol. Soc., pp. 4644-4647, 2008.

[25] C. Zhang and J. H. L. Hansen, "Analysis and Classification of Speech Mode : Whispered through Shouted," pp. 1-4, 2007.

[26] M. Davy and S. Rossignol, "Improved One-Class Svm Classifier For Sounds," pp. 117-122, 2007.

[27] P. C. Schön, B. Puppe, and G. Manteuffel, "Automated recording of stress vocalisations as a tool to document impaired welfare in pigs," Anim. Welf., vol. 13, no. 2, pp. 105-110, 2004.

[28] M. Vacher and D. Istrate, "Sound detection and classification through transient models using wavelet coefficient trees," pp. 1171-1174, 2004.

[29] E. R. Siebert and L. A. Parr, "A Structural and Contextual Analysis of Chimpanzee Screams," vol. 109, pp. 104-109, 2003.

[30] N. E. O. Connor, J. Kuklyte, and P. Kelly, "Anti-social Behavior Detection in Audio-Visual Surveillance Systems," no. June, pp. 40-41, 2009.
[31] R. A. Breguet, "Automatic Sound Detection And Recognition For Noisy Environment," 2000.

[32] D. Lyon, Surveillance studies : an overview. Polity, 2007.

[33] S. Lefèvre and N. Vincent, "A two level strategy for audio segmentation,” Digit. Signal Process. A Rev. J., vol. 21, no. 2, pp. 270277, Mar. 2011.

[34] T. Cover and P. Hart, "Nearest neighbor pattern classification," IEEE Trans. Inf. Theory, vol. 13, no. 1, pp. 21-27, Jan. 1967.

[35] Lie Lu, Hong-Jiang Zhang, and Hao Jiang, "Content analysis for audio classification and segmentation," IEEE Trans. Speech Audio Process., vol. 10 , no. 7, pp. 504-516, Oct. 2002.

[36] N. Bhatia and Vandana, "Survey of Nearest Neighbor Techniques," Jul. 2010.

[37] I. Rojek and M. Jagodziński, "Hybrid Artificial Intelligence System in Constraint Based Scheduling of Integrated Manufacturing ERP Systems," Springer, Berlin, Heidelberg, 2012, pp. 229-240.

[38] D. Turnbull and C. Elkan, "Fast recognition of musical genres using RBF networks," IEEE Trans. Knowl. Data Eng., vol. 17, no. 4, pp. 580584, Apr. 2005.

[39] C. W. Tao, "A reduction approach for fuzzy rule bases of fuzzy controllers," IEEE Trans. Syst. Man Cybern. Part B, vol. 32, no. 5, pp. 668-675, Oct. 2002.

[40] A. Temko, D. Macho, and C. Nadeu, "Fuzzy integral based information fusion for classification of highly confusable non-speech sounds," Pattern Recognit., vol. 41, no. 5, pp. 1814-1823, May 2008.

[41] T. Giannakopoulos, D. Kosmopoulos, A. Aristidou, and S. Theodoridis, "Violence Content Classification Using Audio Features," Springer, Berlin, Heidelberg, 2006, pp. 502-507.

[42] P. Prodanov and A. Drygajlo, "Bayesian networks based multi-modality fusion for error handling in human-robot dialogues under noisy conditions," Speech Commun., vol. 45, no. 3, pp. 231-248, Mar. 2005.

[43] K. Daoudi, D. Fohr, and C. Antoine, "Dynamic Bayesian networks for multi-band automatic speech recognition," Comput. Speech Lang., vol. 17, no. 2-3, pp. 263-285, Apr. 2003.

[44] R. A. Fisher, "The Use Of Multiple Measurements In Taxonomic Problems," Ann. Eugen., vol. 7, no. 2, pp. 179-188, Sep. 1936.

[45] V. N. Vapnik, "Statistical learning theory NewYork," 1998.

[46] S. Sharma and R. L. Yadav, "Comparative Study of Kmeans and Robust Clustering,” Int. J. Adv. Comput. Res., vol. 3, no. 12, 2013.

[47] T. Andreassen, A. Surlykke, and J. Hallam, "Semi-automatic long-term acoustic surveying: A case study with bats," Ecol. Inform., vol. 21, pp. 13-24, May 2014.

[48] L. Kaufman and P. J. Rousseeuw, Finding groups in data: an introduction to cluster analysis. Wiley-Interscience, 1990.

[49] B. Larsen and C. Aone, "Fast and effective text mining using linear-time document clustering," in Proceedings of the fifth ACM SIGKDD international conference on Knowledge discovery and data mining KDD '99, 1999, pp. 16-22.

[50] L. E. Baum and T. Petrie, "Statistical Inference for Probabilistic Functions of Finite State Markov Chains," The Annals of Mathematical Statistics, vol. 37. Institute of Mathematical Statistics, pp. 1554-1563.

[51] J. Principe, N. Euliano, and W. Lefebvre, Neural and adaptive systems: fundamentals through simulations, Vol.672. New York: Wiley, 2000. 